\title{
Influence of a magnetic field on the nematic phase of hard colloidal platelets
}

\author{
D. van der Beek, ${ }^{1, *}$ P. Davidson, ${ }^{2}$ H. H. Wensink, ${ }^{3}$ G. J. Vroege, ${ }^{1}$ and H. N. W. Lekkerkerker ${ }^{1}$ \\ ${ }^{1}$ Van't Hoff Laboratory for Physical and Colloid Chemistry, Utrecht University, Padualaan 8, 3584 CH Utrecht, The Netherlands \\ ${ }^{2}$ Laboratoire de Physique des Solides, UMR 8502 CNRS, Université Paris-Sud, Bâtiment 510, 91405 Orsay, France \\ ${ }^{3}$ Center for Process Systems Engineering, Department of Chemical Engineering and Chemical Technology, Imperial College London, \\ South Kensington Campus, London SW7 2AZ, United Kingdom
}

(Received 16 May 2007; revised manuscript received 15 January 2008; published 28 March 2008)

\begin{abstract}
We report on a study of the influence of a magnetic field on the nematic phase and the isotropic-to-nematic (IN) phase transition of hard colloidal gibbsite platelets. We find direct visual evidence of a shift of the IN transition to lower concentrations due to the magnetic field. The nematic phase readily aligns when contained between two parallel flat glass walls, with homeotropic orientation. This well-defined surface anchoring enables a measurement of the bend Frederiks transition, yielding the bend elastic constant of a nematic phase of hard disks as $K_{3}=7 \times 10^{-14} \mathrm{~N}$. By applying a rotating magnetic field on the nematic phase, we observe the bend-splay Frederiks transition, visible as a spatially periodic transient pattern with a wavelength that depends on sample thickness and field strength. Following a linear stability analysis we are able to account for this dependence in a qualitative way. Moreover, the rotating magnetic field enables us to render the nematic phase single domain, with well-defined director orientation.
\end{abstract}

DOI: 10.1103/PhysRevE.77.031708

PACS number(s): 64.70.M-, 82.70.Dd, 61.30.Gd

\section{INTRODUCTION}

It is well-known that suspensions of hard anisotropic particles, i.e., rod- or plate-like, show the phase transition from a disordered isotropic (I) to a nematic $(\mathrm{N})$ phase, which is characterized by long-range orientational order. The earliest experimental reports date back to the 1920s and 1930s [1], while a substantial theoretical contribution was made by Onsager in the 1940s [2], in which he explained the transition as due to purely repulsive interactions. Later, it was found that hard anisotropic particles also show liquid crystalline phases with translational order. By computer simulation, the smectic [3] and columnar phases [4] were revealed for rods and plates, respectively. The first experimental system to show the full range of these phases for platelets, i.e., isotropic, nematic, and columnar, is the colloidal suspension of sterically stabilized gibbsite $\left[\gamma-\mathrm{Al}(\mathrm{OH})_{3}\right]$ platelets $[5,6]$, developed a decade ago. It has proven to be a very versatile model system of hard disks, as it is easily perturbed by external gravitational [7] or surface [8,9] fields. Moreover, we have recently reported the magnetic-field-induced orientational order in the isotropic phase, measured by birefringence and small-angle x-ray scattering [10]. In this paper, we report results about the influence of a magnetic field on the nematic phase.

In the first part of this paper, we will study the isotropicnematic (IN) phase transition, with and without an external magnetic field. As the IN phase transition involves a gain in orientational order, one may intuitively expect that the influence of an external aligning field will be beneficial to this ordering. This indeed appears to be the case. Khokhlov and Semenov, as well as Varga and co-workers have shown that

\footnotetext{
*Present address: Albemarle Catalysts Company B.V., Nieuwendammerkade 1-3, 1022 AB Amsterdam, The Netherlands. david.vanderbeek@albemarle.com
}

rods display the IN phase transition at lower density upon increasing the external field strength [11]. For platelike particles their results are valid as well, albeit only qualitatively. So far, experimental evidence for a shift of the IN transition of colloidal systems in an external magnetic field is limited: for rods only indirect evidence exists [12], while for plates no such observations have been made, despite some studies of diamagnetic colloidal plates in a magnetic field $[13,14]$. Here, we present clear visual evidence for a shift in the IN phase transition due to the magnetic field, in our suspension of colloidal gibbsite platelets.

There are two possible scenarios for the alignment of rods or plates in an external magnetic field $[11,15,16]$. Depending on the sign of the diamagnetic susceptibility anisotropy $\Delta \chi$, the particles align their axes either parallel or perpendicular to the field, which has implications for the phase behavior. In Fig. 1, we have sketched the phase diagrams for both scenarios. For positive $\Delta \chi$, the particles' axes align parallel to the field and there will be a transition from a paranematic (field-aligned isotropic phase) to a nematic phase, both of

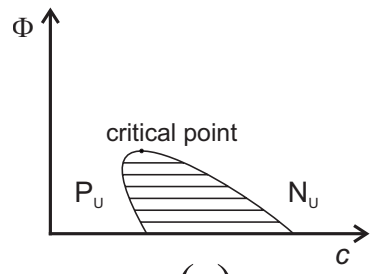

(a)

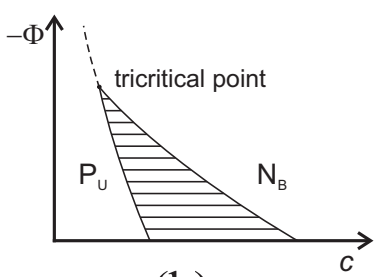

(b)
FIG. 1. Sketches of the phase diagram of rods or platelets in an external field, after Refs. [11,15], (a) for positive and (b) for negative values of the field interaction parameter $\Phi \propto \Delta \chi B^{2}$, a measure of the magnetic energy per particle. The solid horizontal lines denote tie lines, $c$ denotes the concentration. $\mathrm{P}$ and $\mathrm{N}$ refer to the paranematic and nematic phases, respectively, and the subscripts $\mathrm{U}$ (uniaxial) and B (biaxial) denote the phase symmetry. 
uniaxial symmetry, see Fig. 1(a). For negative $\Delta \chi$, the particles tend to align perpendicular to the field: the transition becomes uniaxial-biaxial and the shape of the biphasic region changes, see Fig. 1(b).

The colloidal gibbsite platelets studied here have a negative $\Delta \chi[10]$. On applying a static magnetic field this will result in the latter type of ordering in our suspension. The order parameter $S \equiv\left\langle P_{2}(\cos \theta)\right\rangle$, where $\theta$ is the angle between the particle axis and the magnetic field, will have a negative value, both for the paranematic phase $\left(-\frac{1}{2} \leq S \leq 0\right)$ and the biaxial nematic phase $(-1 \leq S \leq 0)$. For a rotating field, it makes sense to define $\theta$ as the angle between particle axis and the rotation axis of the field, resulting in positive values of the order parameter. Moreover, as will be shown, with a rotating field it is possible to obtain a nematic single domain with well-defined orientation.

In the second part of this paper, we describe the nematic phase with and without magnetic field. In particular, we focus on the magnetic-field-induced distortion in a thin nematic layer between two flat walls, known as the Frederiks [17] transition $[18,19]$. The origin of this transition is the competition between the surface anchoring of the nematic phase and the volume effect due to the magnetic field opposing this orientation. Because of the elasticity of the nematic liquid crystalline phase, as governed by the elastic moduli $K_{i}$ $(i=1,2,3)$, a certain threshold field is required to induce the transition. By carefully measuring this threshold for samples of varying thickness, we have been able to determine the bend elastic constant $K_{3}$ of our nematic phase. Earlier observations of the Frederiks transition in colloidal suspensions comprise rodlike $[20,21]$ and disklike particles [13]. The latter system, composed of disklike micelles with negative $\Delta \chi$, is completely analogous to the one under consideration here. The effect of a rotating magnetic field, mentioned above, is important for the Frederiks transition as well. From geometric considerations it appears that with a static field a uniform bend transition is induced. Using a rotating field, we bring about a nonuniform bend-splay transition involving very typical transient periodic patterns, as first observed by Carr [22] and analyzed by Guyon, Meyer, and Salan [23] using a linear stability analysis.

Our paper is organized in the following way. In Sec. II, we describe the synthesis and characterization of our suspension of sterically stabilized gibbsite platelets. In Sec. III, we study the IN phase separation in zero field and in a magnetic field. Then, in Sec. IV, we discuss some characteristics of the nematic phase in zero field, before moving on to the nematic phase studied in a magnetic field in Sec. V. There, we investigate the uniform bend as well as the transient bend-splay Frederiks transition. We end our paper with a summary and conclusions in Sec. VI.

\section{SAMPLE PREPARATION AND CHARACTERIZATION}

We synthesized hexagonal colloidal gibbsite $\left[\gamma-\mathrm{Al}(\mathrm{OH})_{3}\right]$ platelets grafted with end-functionalized polyisobutene and dispersed in toluene, following a method developed a few years ago [24]. Thus a model system of hard platelets is obtained, showing the liquid crystal phase transi- tions predicted for hard-disk fluids [4-6,8]. Transmission electron microscopy and atomic force microscopy were used to determine the average diameter $\langle D\rangle$ and thickness $\langle L\rangle$ of the dry particle core. The diameter of a platelet is measured as the average of the three corner-to-corner distances and the estimated thickness may contain a contribution of the collapsed steric stabilizer. We found $\langle D\rangle=232 \mathrm{~nm}$ and $\langle L\rangle$ $=13 \mathrm{~nm}$ and a polydispersity of about $20 \%$ in both dimensions. In solution, the thickness of the sterically stabilizing polyisobutene brush is estimated to be 2 to $3 \mathrm{~nm}$, yielding effective dimensions of $D_{\text {eff }}=237 \mathrm{~nm}$ and $L_{\text {eff }}=18 \mathrm{~nm}$.

The dispersion of gibbsite platelets shows the first-order isotropic-to-nematic phase transition, with coexistence of the two phases between volume fractions $\phi_{\mathrm{I}}=0.238$ and $\phi_{\mathrm{N}}=0.255$. Volume fractions were calculated from the mass concentration determined by drying a known amount of dispersion under nitrogen at $85^{\circ} \mathrm{C}$ to constant weight and the particle mass density $\rho_{\mathrm{p}}=1.7 \mathrm{~g} / \mathrm{ml}[5,25]$. Samples were concentrated by centrifugation and removal of supernatant solvent, or diluted by adding pure solvent, while keeping track of the actual volume fraction by weighing.

\section{ISOTROPIC-NEMATIC PHASE SEPARATION}

\section{A. In zero field}

As was observed earlier, IN phase separation in this specific colloidal model system takes place in 6-12 h [5,6]. In comparison with atomic systems, whose constituents are orders of magnitude smaller, this is a relatively large time scale [26]. Here, we exploit this feature by studying the IN phase separation process with an ordinary digital camera.

\section{Experiment}

A spectrophotometric cell (light path $2.00 \mathrm{~mm}$, volume $\sim 700 \mu \mathrm{l})$ was filled with a sample of the suspension, at $\phi=0.25$, in the IN biphasic gap. After thorough homogenization, the sample was left undisturbed to phase separate. During this process the sample was observed between crossed polarizers, while digital images, with a resolution of $640 \times 480$ pixels were automatically recorded each $125 \mathrm{~s}$ for a total time of $24 \mathrm{~h}$, using a Nikon Coolpix 995 charge coupled device $(\mathrm{CCD})$ camera.

\section{Results and discussion}

The characteristic events in the phase separation process are depicted in Fig. 2. In Fig. 2(a) (right after homogenization) birefringence is visible throughout the sample, still bearing the marks of the flow of the entire sample within the cell. During the first $40 \mathrm{~min}$, the birefringent patches seem to move through the sample, as in Fig. 2(b), and only after this "waiting time" a visible separation process seems to start. The low-density droplets of isotropic phase migrate upwards, while the high-density nematic phase comes down. The sample develops lanes in which the material travels up or down, which seems to facilitate the separation of the two phases, see Figs. 2(c) and 2(d). The interface between the two phases is first only ill-defined [Fig. 2(c)], but becomes more pronounced during the separation process [Fig. 2(d)]. It 


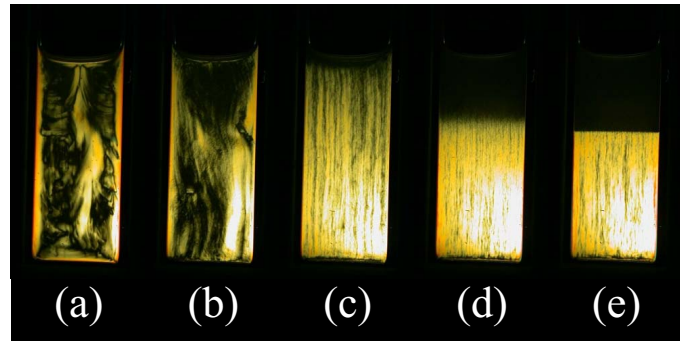

FIG. 2. (Color online) Snapshots of the IN phase separation process, taken (a) just after homogenization, after (b) $26 \mathrm{~min}$, (c) 2 h, (d) $7 \mathrm{~h}$, and (e) $10 \mathrm{~h}$. After a "waiting time" of about $40 \mathrm{~min}$ the actual separation of the phases starts. The interface, which is first only ill-defined, gradually settles to become very sharp.

then descends at a constant velocity of about $0.3 \mu \mathrm{m} / \mathrm{s}$ to yield a sharp interface after $10 \mathrm{~h}$, see Fig. . The final striped texture of the nematic phase has been observed earlier in our suspensions of platelets (see, e.g., Ref. [27]) and is clearly caused by the preceding phase separation process. Lane formation has been observed earlier in phase separating colloidal suspensions [26,28]. However, in the present case, the relevant time scales (like, for example, the "waiting time" and the time that the lanes persist) are even larger. It would therefore be interesting to use the present colloidal suspension of hard platelets in a more thorough study of such phase separation phenomena.

In weeks, the striped texture of the nematic phase disappears to yield a single domain nematic phase, oriented by the sample walls and the IN interface. Close inspection of the interface near the wall reveals a capillary rise. In a previous paper we have used this capillary rise to calculate the IN interfacial tension and found good agreement with density functional calculations and computer simulations [29].

\section{B. In nonzero field}

We now turn to the investigation of the effect of the magnetic field on the location of the IN phase transition. From theory [11] it follows that, at fixed overall volume fraction in the biphasic IN gap, the relative amount of nematic phase should increase with increasing field strength. Whether this is indeed the case is tested in this section, and we use both static and rotating magnetic fields.

\section{Experiment}

We prepared a sample of the dispersion in a glass cell (inner cross section $2 \times 4 \mathrm{~mm}^{2}$ ) at a volume fraction of $\phi$ $=0.248$, in the IN biphasic regime. We studied its phase behavior as a function of overall volume fraction and magnetic field strength. The overall volume fraction, ranging from $\phi$ $=0.238$ to 0.248 , was varied as outlined in Sec. II. The magnetic field was generated with a Bruker BE25v electromagnet, equipped with large flat pole shoes producing a very homogeneous magnetic field in the sample volume. Field strengths ranged from $B=0-2.5 \mathrm{~T}$ and were monitored with a LakeShore 421 Gaussmeter. For each measurement, we thoroughly homogenized the sample outside the magnet and

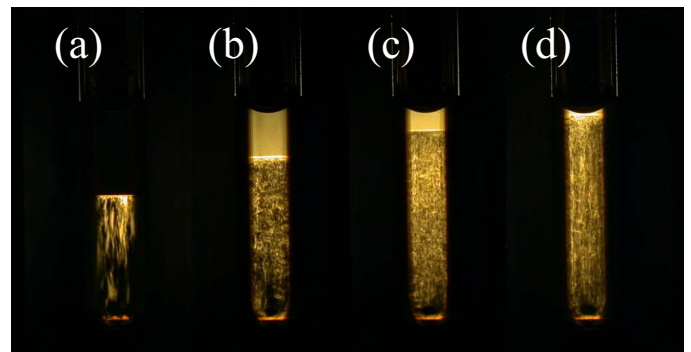

FIG. 3. (Color online) The IN phase separation, (a) in zero field, and in a magnetic field of squared intensities (b) 2.0, (c) 4.0, and (d) $6.0 \mathrm{~T}^{2}$, at a constant overall volume fraction $\phi=0.248$. The magnetic field was directed horizontally, the crossed polarizers made an angle of $45^{\circ}$ with the vertical. We note that the phase separation with or without the external magnetic field yields different textures of the nematic phase. This might point to different phase separation mechanisms, e.g., spinodal decomposition vs nucleation and growth.

immediately suspended it between the poles of the magnet that was already at the intended field strength. The sample was left to phase separate in the field, which took at least 1 day, hence significantly longer than in zero field. The sample was visually inspected between crossed polarizers (both making an angle of $45^{\circ}$ with the vertical) and photographic images were taken with a Nikon Coolpix 995 CCD camera.

In addition to the described experiments with a static magnetic field, we investigated the IN phase behavior in a rotating field. To that end, the sample was set on a rotation stage mounted between the magnet poles. We rotated the sample at a very low rate of $1 / 6 \mathrm{~Hz}$, slow enough to avoid centrifugal forces in the sample, but fast enough to overcome the (collective) rotational diffusion in the nematic phase.

\section{Results and discussion}

Figure 3 depicts photographs of the phase separated sample at $\phi=0.248$ and with increasing magnetic field strength. There are a few striking features in this figure. First, we note that the isotropic phase appears completely black in zero field, but acquires a magnetic-field-induced birefringence on increasing field strengths. Exactly this phenomenon has been studied in our previous paper [10]. Second, we find that the texture of the nematic phase at nonzero field is not striped, like in zero field (see Sec. III A), but grainy. As the texture of the nematic phase appears to be directly caused by the phase separation process, this might point to two different phase separation mechanisms, i.e., spinodal decomposition vs nucleation and growth. Most importantly, the amount of nematic phase increases with field strength, from about $60 \%$ in zero field up to almost $100 \%$ at $B^{2}=6.0 \mathrm{~T}^{2}$. (For the rotating field, we find the same relative amounts of nematic phase for the same field strengths.)

In Fig. 4 we present all the primary data for the phase separation experiments and the resulting paranematic and nematic binodal points, including error bars, under the assumption that the lever rule applies. This is strictly speaking only valid for monodisperse samples, and our data points at 


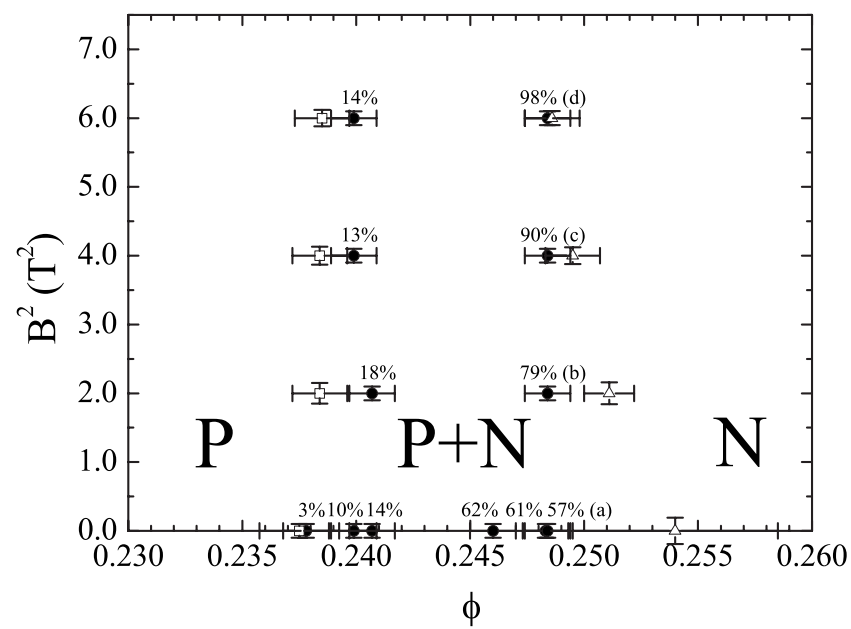

FIG. 4. Phase diagram of the suspension of hard colloidal gibbsite platelets in a magnetic field. The measured, primary data points (or state points, black dots) are labeled with the relative amount of nematic phase $(\% N)$. From these points, the paranematic (open squares) and nematic (open triangles) limits of stability (or binodal points) are extrapolated using the lever rule. The resulting error is indicated with the horizontal bars. The state points indicated by (a)-(d) pertain to the samples depicted in Fig. 3. P and $\mathrm{N}$ indicate the location of the paranematic and nematic phase, respectively, while the biphasic region in the field is indicated with $\mathrm{P}+\mathrm{N}$.

zero field indeed indicate a slight deviation from the lever rule due to polydispersity. Let us now discuss the uncertainties in the primary data presented in Fig. 4, i.e., the volume of the nematic phase as a percentage of the total volume $(\% N)$ at different overall concentrations and magnetic field strengths. First, there are the errors in the overall volume fractions, typically \pm 0.001 , which may be due to slight evaporation of the solvent and weighing errors. The error in the magnetic field strength squared is about $\pm 0.1 \mathrm{~T}^{2}$; its source may be the field strength measuring equipment. We estimate the error in $\% N$ at $\pm 2 \%$, and attribute this to the uncertainty in the measurement of the heights of the phases and the possible variation in $\% N$ itself. Our data in Fig. 4 clearly show that the biphasic gap narrows on increasing field strength, in agreement with theory [11].

\section{NEMATIC PHASE}

We will now investigate the nematic phase in equilibrium. To that end, we use microscopy to visualize the textures of the nematic phase. In addition, we employ x-ray scattering to determine the orientation and nematic order parameter of the nematic phase.

\section{A. Optical microscopy}

\section{Experiment}

In order to obtain a nematic phase of the platelet dispersion, we started from a sample of the dispersion in the IN biphasic gap. After 1 day, the sample had phase separated, yielding about $90 \%$ isotropic phase and $10 \%$ nematic phase.

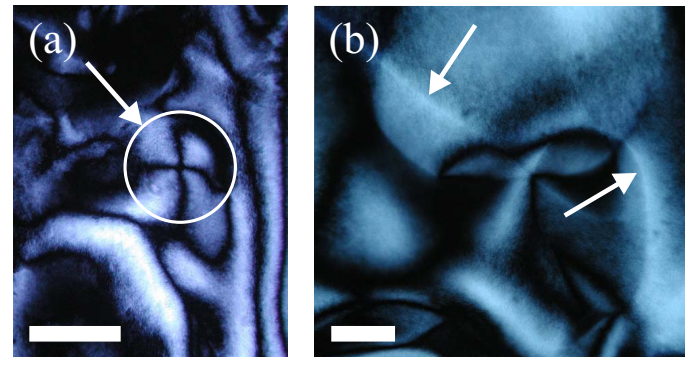

FIG. 5. (Color online) The nematic phase of sterically stabilized colloidal gibbsite platelets shows defects typical for a nematic phase, like the (a) four-brush defect and (b) disclination lines indicated by the white arrows. The scale bars denote 100 and $500 \mu \mathrm{m}$, respectively.

Both phases were extracted; the nematic phase was used here. The volume fraction of the nematic phase amounted to $\phi_{\mathrm{N}}=0.27$, which corresponds to a number density of $\rho$ $=N / V=4.1 \times 10^{20} \mathrm{~m}^{-3}$. We attribute the slightly higher nematic coexistence concentration to fractionation effects. Several samples of the nematic phase were prepared in flat optical capillaries (VitroCom, $\mathrm{NJ}$ ) with internal dimensions of $50 \mu \mathrm{m} \times 0.5 \mathrm{~mm} \times 10 \mathrm{~cm}$ and $300 \mu \mathrm{m} \times 3.0 \mathrm{~mm}$ $\times 10 \mathrm{~cm}$, by sucking the nematic phase into the capillary using vacuum. The capillaries were subsequently flamesealed to avoid evaporation of the solvent. The samples were investigated with polarized light microscopy (PLM), here, and in the remainder of the paper, with an Olympus BX51 microscope equipped with a Canon Camedia C-3030 CCD camera.

\section{Results and discussion}

Freshly prepared samples of the nematic phase display topological defects that are very typical, like a four-brush defect (strength $m= \pm 1$ ) and disclination lines, see Fig. 5. Because the capillaries are relatively thin, the defects anneal on a time scale of several days to yield single domain nematic phases with homeotropic surface anchoring. For thicker samples it takes weeks, as mentioned in Sec. III A. Such nematic single domains were further used to study the Frederiks transition, as described in Sec. V.

\section{B. X-ray scattering}

\section{Experiment}

X-ray diffraction experiments were performed with a laboratory setup described in Ref. [30]. The beam $\left(\lambda_{\mathrm{Cu}-K \alpha}\right.$ $=1.541 \AA$ ) delivered by a Rigaku rotating-anode generator was focused and filtered with mirror X-ray optics. A sample of the nematic phase was held in a cylindrical Lindemann glass capillary (diameter $1 \mathrm{~mm}$ ), which was rotated along its vertical axis in a horizontal $1 \mathrm{~T}$ magnetic field, see Fig. 6(a). The scattered $\mathrm{x}$-rays were detected on imaging plates and typical exposure times ranged from 1 to $20 \mathrm{~h}$. The smallangle scattering was recorded with a sample-detector distance of $1.05 \mathrm{~m}$ and vacuum tubes were inserted between the sample and the detector in order to avoid parasitic air scat- 


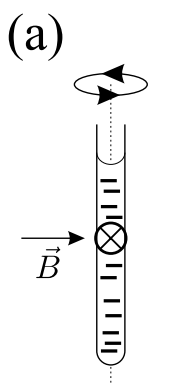

(b)

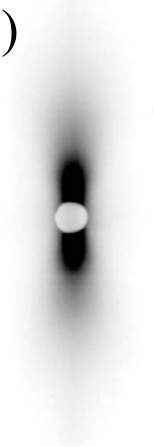

(c)

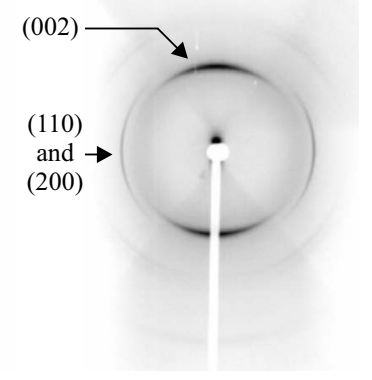

FIG. 6. X-ray scattering patterns of the nematic phase. (a) The nematic phase was contained in a cylindrical Lindemann capillary that made right angles with the magnetic field $\vec{B}$ of $1 \mathrm{~T}$ and the $\mathrm{X}$-ray beam (perpendicular to the plane of the figure, as indicated by the crossed circle). The sample was rotated around its vertical axis to create a single domain nematic phase during recording of (b) the small-angle and (c) the wide-angle scattering patterns; both are shown on a linear intensity scale. Crystallographic peaks of gibbsite are indicated by their Miller indices in (c).

tering. The scattering at wide angles was recorded with a sample-detector distance of $62 \mathrm{~mm}$, directly in air.

\section{Results and discussion}

As expected, the small-angle X-ray scattering (SAXS) signal, see Fig. 6(b), of the nematic phase rotated in the horizontal magnetic field is very anisotropic, which demonstrates the good alignment of the platelets. The diffuse scattering extends much more in the vertical direction, showing that the disks lie horizontal, with their normal parallel to the vertical capillary axis. This observation is consistent with previous experiments with the isotropic phase of the gibbsite suspensions [10]. It proves that the nematic phase has a negative anisotropy of diamagnetic susceptibility $(\Delta \chi<0)$ and the sample must be rotated in the field in order to reach full alignment. The decay of the scattered intensity with scattering vector modulus $|\vec{q}|\left(|\vec{q}|=q=4 \pi \sin \vartheta / \lambda_{\mathrm{Cu}-K \alpha}\right.$, where $2 \vartheta$ is the scattering angle and $\lambda_{\mathrm{Cu}-K \alpha}$ the wavelength of our setup, see above) is monotonous, see Fig. 7. Only a small shoulder, pointing to faint liquidlike positional order, is observed around $q=0.2 \mathrm{~nm}^{-1}$. The absence of a real correlation peak arising from interparticle interferences allows us to use a simplified model to derive the nematic order parameter $S$ from the SAXS pattern [31]. This data treatment, already used for laponite clay suspensions, assumes that the scattering is only determined by the particle form factor and by the orientational distribution function which is chosen here in the Maier-Saupe form. The value obtained, $S=0.85 \pm 0.05$, is very large, illustrating the pronounced orientational order in the nematic phase.

The wide-angle scattering of the same aligned nematic sample of gibbsite suspension, see Fig. 6(c), reveals sharp diffraction arcs that arise from the internal crystallographic structure of gibbsite platelets. Gibbsite nanoparticles crystallize in the monoclinic (pseudohexagonal) $P 2_{1} / n$ space group with the $c$ (pseudo-C6) axis along their normal [32]. The

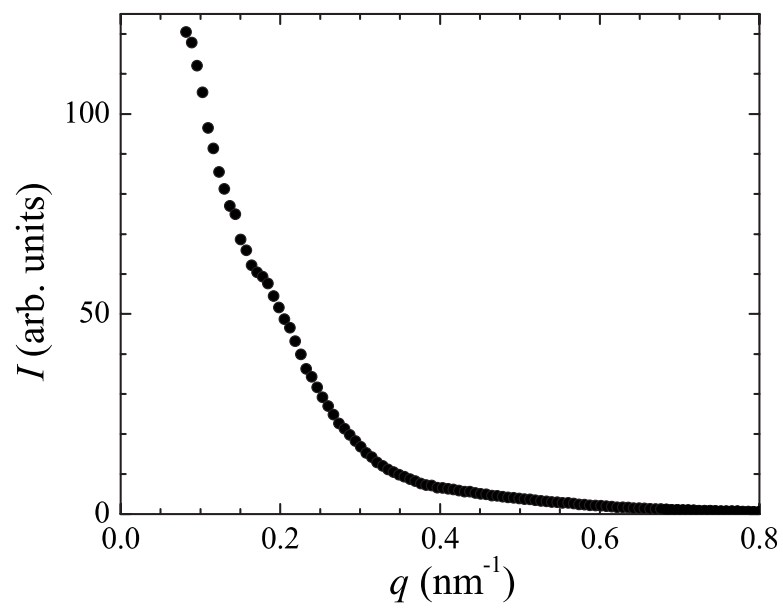

FIG. 7. Intensity profile of the SAXS pattern in Fig. 6(b), taken along the vertical. A shoulder is just visible around $q=0.2 \mathrm{~nm}^{-1}$, pointing to slight liquidlike ordering of the platelets in the nematic phase.

meridional arc located along the vertical direction is due to the (002) reflection, which is broadened beyond instrumental resolution by the limited particle thickness. The equatorial arc represents both the (200) and the (110) reflections that correspond to very similar lattice spacings. The location and rather small extension of the (002) reflection confirm that the platelets are well-aligned with their normal parallel to the capillary main axis. The nematic order parameter can also be inferred from the azimuthal spread of the (002) reflection by using a well-documented procedure [33]. The value obtained in this way, $S=0.80 \pm 0.05$, is in good agreement with the previous one derived from the SAXS pattern. Similar agreement was observed in a combined SAXS-wide angle $\mathrm{X}$-ray scattering (WAXS) study of the nematic phase of rodlike colloidal particles [34].

\section{FREDERIKS TRANSITION}

In this section two types of experiments will be performed, henceforth called A and B and sketched in Fig. 8. In

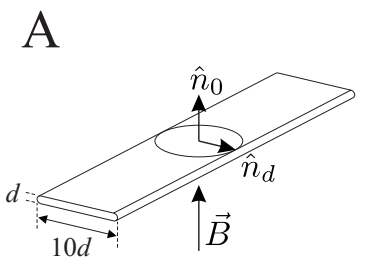

B

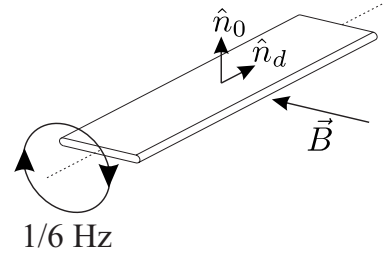

FIG. 8. Geometry of the two types of experiments performed. In experiment $\mathrm{A}$, the magnetic field $\vec{B}$ is applied perpendicular to the sample wall, inducing a uniform bend Frederiks transition, while in experiment $\mathrm{B}$ a nonuniform bend-splay transition is induced by rotating the sample around its long axis. $\hat{n}_{0}$ and $\hat{n}_{d}$ indicate director field orientations before and after the transition, respectively, while the circle in A indicates rotational degeneracy. 
experiment $\mathrm{A}$, the uniform bend Frederiks transition is induced that yields the bend elastic constant of the nematic phase, while in experiment $\mathrm{B}$ the nonuniform bend-splay transition is probed, showing a very typical transient periodic stripe pattern.

\section{A. Uniform bend transition}

\section{Experiment}

We prepared nematic samples in flat optical capillaries with thickness $d$ ranging from 20 to $100 \mu \mathrm{m}$, width $10 d$, and length $\sim 70 \mathrm{~mm}$ in the same fashion as in Sec. IV A. The topological defects present after preparation disappeared over several days to yield a homeotropically anchored single domain nematic phase. (Thicker samples of 200 and $300 \mu \mathrm{m}$ were also prepared, but not used because hydrodynamic flow could not be avoided.) The preferential homeotropic orientation of the platelets, i.e., with their normal $\hat{n}$ perpendicular to the glass walls, is understood as due to excluded volume effects and has been discussed before $[8,9,35]$. Before each measurement, each sample was placed in a magnetic field (of $\sim 1 \mathrm{~T}$ ) oriented parallel to the capillary walls overnight, as to reach the well-defined initial homeotropic alignment faster in between successive experiments.

The experimental procedure consisted of inserting a sample in a homemade permanent magnet with tunable field strength, such that the field was directed perpendicular to the sample wall, see Fig. 8, while keeping the sample horizontal. After keeping the sample $1 \mathrm{~h}$ in the field, it was investigated by PLM in situ. This was repeated for increasing field strengths from 5 to $500 \mathrm{mT}$. We determined the critical field strength $B_{\mathrm{c}}$ for the onset of the bend transition when the first signs of birefringence appeared.

\section{Results and discussion}

As inferred from the direction of the initial and final director fields, we conclude that the bend transition is probed and that the corresponding expression for the critical field strength $B_{\mathrm{c}}$ is given by [19]

$$
B_{\mathrm{c}}=\frac{\pi}{d} \sqrt{\frac{K_{3}}{\rho|\Delta \chi|}},
$$

where $d$ is the sample thickness, $K_{3}$ the bend elastic constant, $\rho$ the particle number density, and $\Delta \chi$ the diamagnetic susceptibility anisotropy per particle. In Fig. 9 we plot the (reciprocal) critical field strength versus sample thickness. Our data pass through the origin (to within experimental error), indicating that the initial homeotropic anchoring is strong. (For so-called weak anchoring, a shift of the curve to the left is to be expected, requiring incorporation of a virtual sample thickness $>d$.) A one-parameter fit through our data is therefore justified, its slope equaling $\sqrt{\rho|\Delta \chi| / \pi^{2} K_{3}}$, see Eq. (1). We are now able to calculate the bend elastic constant of the nematic phase by taking $\Delta \chi=-10^{-22} \mathrm{~J} / \mathrm{T}^{2}[10]$ and $\rho=4.1$ $\times 10^{20} \mathrm{~m}^{-3}$ to obtain $K_{3}=7 \pm 1 \times 10^{-14} \mathrm{~N}$.

It would be interesting to compare this value with other work. However, there are no other experiments on the elastic

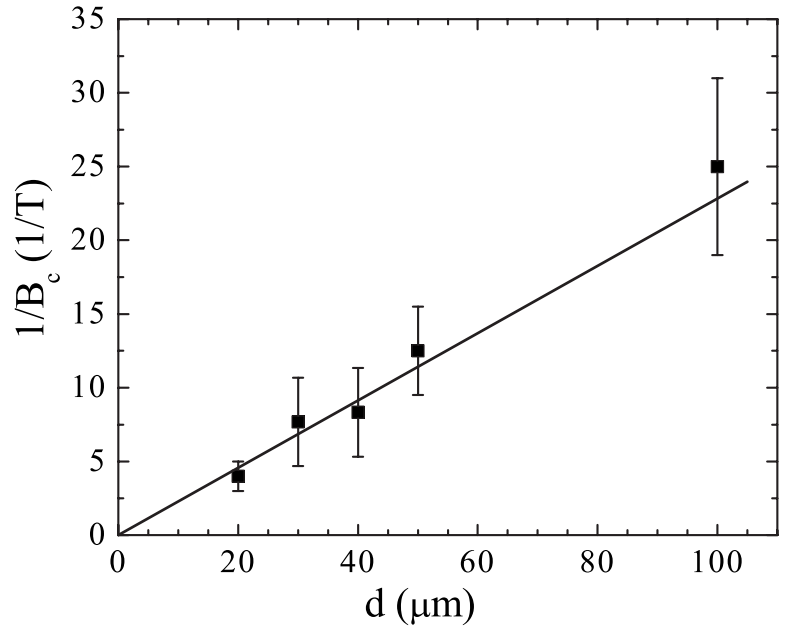

FIG. 9. Reciprocal threshold field strength $1 / B_{\mathrm{c}}$ for the uniform bend Frederiks transition as a function of sample thickness $d$. As our data indicate strong anchoring, we perform a linear oneparameter fit to obtain $K_{3}$ from its slope.

constants of a nematic phase of platelike particles, nor computer simulations and theoretical predictions, so a direct comparison is not possible. We can make a crude comparison with rodlike particles, though. The cholesteric phase of 45 $f d$-virus particles has a twist elastic constant $K_{2} \cong 4$ $\times 10^{-13} \mathrm{~N}[21,36]$, which is of the same order of magnitude.

\section{B. Nonuniform bend-splay transition}

\section{Experiment}

Measurements of the nonuniform bend-splay Frederiks transition in our nematic phase, experiment B, were performed on 50- and $100-\mu \mathrm{m}$ thick samples, prepared as described in Sec. V A. Before each measurement, the sample was carefully checked to be completely homeotropic. The sample was rotated in the homemade magnet around its long axis at an angular speed of about $10 \mathrm{rpm}(1 / 6 \mathrm{~Hz})$ for up to $10 \mathrm{~min}$, at magnetic field strengths ranging from 0.2 to $1 \mathrm{~T}$. The samples were inspected with in situ-PLM at regular intervals during the procedure and micrographs were taken. Flow of the nematic phase in the capillary did not occur.

\section{Results and discussion}

Upon rotation, the samples show a striking periodic pattern, analogous to the study by Kuzma on a suspension of disklike micelles [13]. The spatial periodicity ranges typically from 20 to $100 \mu \mathrm{m}$, depending on sample thickness and field strength. At relatively high magnetic field, the periodic pattern appears as early as after one revolution of the sample in the field. In a few minutes, the wavelength $\lambda$ of the pattern slightly increases to reach a saturation value [37]. At much longer times, the stripes disappear and the nematic phase becomes a single domain with its director along the rotation axis. Figure 10 shows an example of a periodic pattern and its development in time. For each measurement, the wavelength of the periodic pattern was obtained by direct measurement of 20-80 fringes. 

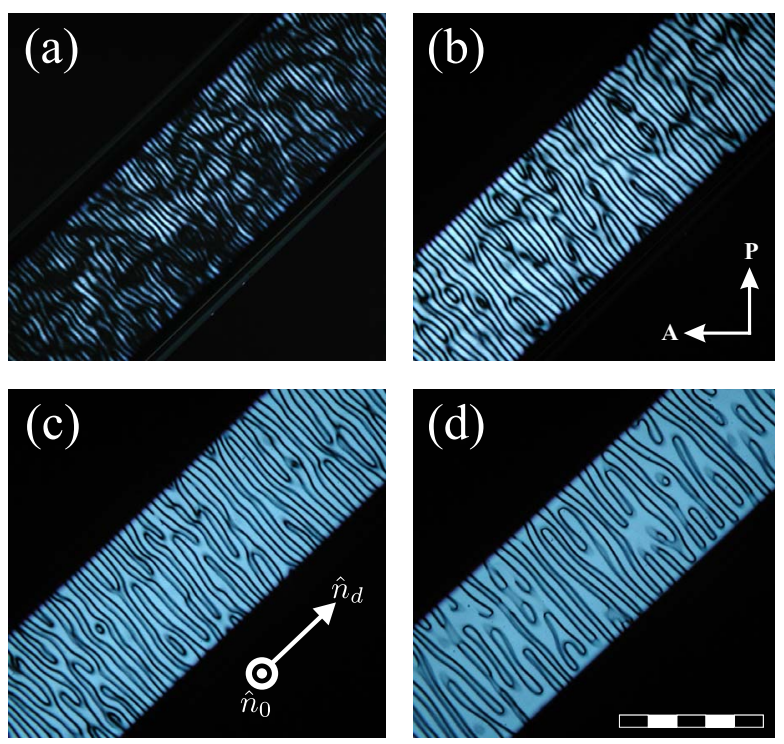

FIG. 10. (Color online) Development in time of the periodic pattern of the nonuniform bend-splay Frederiks transition, in a sample with thickness $d=100 \mu \mathrm{m}$ at $B=560 \mathrm{mT}$. The polarization photographs were taken at (a) $t=5 \mathrm{~s}$, (b) $86 \mathrm{~s}$, (c) $200 \mathrm{~s}$, and (d) 554 s. Polarizer orientations $(A-P)$, scale bar (denoting $1 \mathrm{~mm})$, and initial and final director orientations $\hat{n}_{0}$ and $\hat{n}_{d}$ pertain to all four subgraphs.

The periodic response is due to a strong coupling between fluid flow and director rotation, and the large viscous and elastic anisotropy of the nematic phase. Through a periodic distortion the effective viscosity is reduced and the response speed is increased compared to a uniform distortion. As soon as the rotating field is applied, the nematic phase is in unstable equilibrium and small fluctuations of the director field grow exponentially, with the fastest mode dominating and yielding the observed periodic pattern. In order to analyze this problem, we turn to Fraden and Meyer [38]. Inspired by Kuzma's experiments [13], they performed a linear stability analysis to give an expression for the growth rate $s$ of the dominating mode. In dimensionless units, it is given by

$$
\bar{s}=\frac{\gamma_{1} s}{K_{3}(\pi / d)^{2}}=\frac{(y-1-\kappa x)\left(1+\nu x+\eta x^{2}\right)}{\left(1+\nu x+\eta x^{2}\right)-\alpha(1-\beta x)^{2}},
$$

where $y=\left(B / B_{c}\right)^{2}, \quad \kappa=K_{1} / K_{3}, \quad \alpha=\alpha_{2}^{2} / \gamma_{1} \eta_{c}, \quad \beta=\alpha_{3} / \alpha_{2}, \quad \eta$ $=\eta_{b} / \eta_{c}, \nu=N / \eta_{c}$, and $x=(2 d / \lambda)^{2}$, using standard notations [19]. Unfortunately, this expression does not yield a simple and straightforward solution for the observed wavelength. However, if we assume that $\beta, \eta \gg 1$ [38] and that $x>1$, maximizing $\bar{s}$ with respect to $x$ yields

$$
\left(\frac{B}{B_{\mathrm{c}}}\right)^{2}=\left(1+\frac{\kappa \nu}{\alpha \beta^{2}}\right)+\left(\frac{2 \eta \kappa}{\alpha \beta^{2}}\right) x+\frac{\eta \kappa}{\nu}\left(\frac{\eta}{\alpha \beta^{2}}-1\right) x^{2},
$$

a quadratic relation such as that found for the nonuniform twist-bend Frederiks transition [39]. We plot our experimental data accordingly in Fig. 11 and indeed observe the quadratic(-like) dependence. However, in view of the assumptions made and the relatively large scatter, extraction of material parameters is not possible.

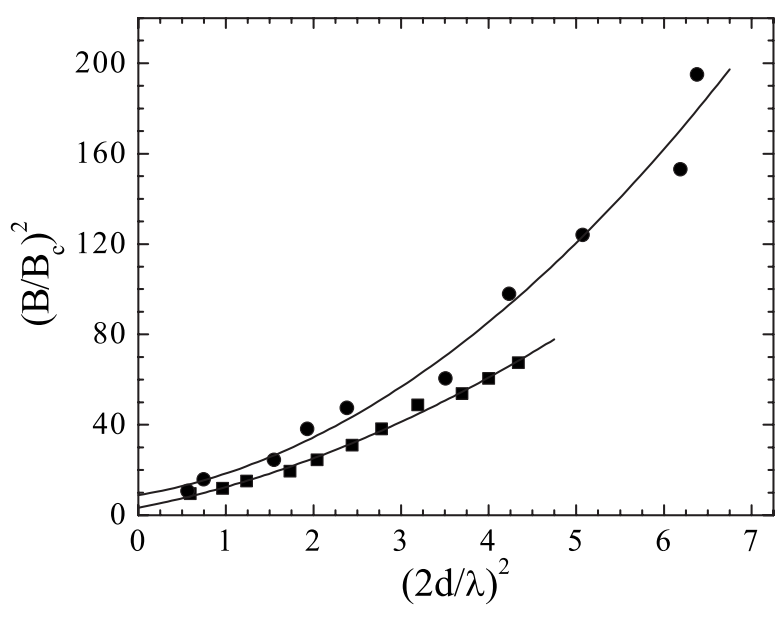

FIG. 11. Experimental data of the nonuniform bend-splay Frederiks transition in the nematic phase. Inspired by Eq. (3) we plot the data as the scaled magnetic field strength squared $\left(B / B_{\mathrm{c}}\right)^{2}$ vs scaled wavelength squared $(2 d / \lambda)^{2}$ for both the $50 \mu \mathrm{m}$ (squares) and $100 \mu \mathrm{m}$ (circles) samples. The lines serve only to guide the eye.

\section{SUMMARY AND CONCLUSIONS}

In this paper, we have studied the nematic phase of hard colloidal gibbsite platelets in an external magnetic field. To that goal, we have first focused on the isotropic-nematic phase transition, first in zero field and then in the magnetic field. During the phase separation process we observe the formation of vertical lanes. The results also seem to indicate that the phase separation mechanism is different with and without the field.

The effect of a magnetic field on the IN phase transition is clear: we find direct visual evidence of a shift of the transition due to the field, as predicted before. As the use of computer simulations in this direction has so far been relatively modest, we suggest to model hard diamagnetic disks and determine the magnetic-field-induced shift of the IN phase transition in computer simulations.

The nematic phase shows very typical characteristics, like topological defects as observed with polarized light microscopy. Because the diamagnetic susceptibility anisotropy $\Delta \chi$ $<0$, rotation of the nematic phase fully aligns the particles to yield a single domain nematic. This is confirmed by small and wide-angle $\mathrm{x}$-ray scattering experiments. Moreover, from the scattering experiments we obtain the nematic order parameter $S=0.85$.

The nematic phase of our platelets contained between two flat walls shows the so-called Frederiks transition, where an initially homeotropic surface anchoring is distorted by a sufficiently high magnetic field. We have been able to locate the threshold field strength for the uniform bend transition, and we have thus determined an elastic constant of a nematic phase of hard disks, i.e., the bend elastic constant $K_{3}=7$ $\times 10^{-14} \mathrm{~N}$.

On applying a magnetic field in a rotating geometry, the bend-splay Frederiks transition is induced, involving transient and spatially periodic patterns. The wavelength of these patterns depends on the magnetic field strength and sample 
thickness and shows qualitative agreement with the linear stability analysis that we performed.

\section{ACKNOWLEDGMENTS}

We thank R. Stannarius for fruitful discussions. The
"Nederlandse Organisatie voor Wetenschappelijk Onderzoek" is acknowledged for funding and the European Marie Curie Training Site Program (Contract No. HPMT-CT-200000047) for making possible the stay of D.B. at the Université Paris-Sud.
[1] H. Zocher, Z. Anorg. Chem. 147, 91 (1925); F. C. Bawden, N. W. Pirie, J. D. Bernal, and I. Fankuchen, Nature (London) 138, 1051 (1936); I. Langmuir, J. Chem. Phys. 6, 873 (1938).

[2] L. Onsager, Phys. Rev. 62, 558 (1942); Ann. N. Y. Acad. Sci. 51, 627 (1949).

[3] D. Frenkel, H. N. W. Lekkerkerker, and A. Stroobants, Nature (London) 332, 822 (1988).

[4] J. A. C. Veerman and D. Frenkel, Phys. Rev. A 45, 5632 (1992).

[5] F. M. van der Kooij and H. N. W. Lekkerkerker, J. Phys. Chem. B 102, 7829 (1998).

[6] F. M. van der Kooij, K. Kassapidou, and H. N. W. Lekkerkerker, Nature (London) 406, 868 (2000).

[7] D. van der Beek, T. Schilling, and H. N. W. Lekkerkerker, J. Chem. Phys. 121, 5423 (2004).

[8] D. van der Beek, A. V. Petukhov, S. M. Oversteegen, G. J. Vroege, and H. N. W. Lekkerkerker, Eur. Phys. J. E 16, 253 (2005).

[9] A. V. Petukhov, D. van der Beek, R. P. A. Dullens, I. P. Dolbnya, G. J. Vroege, and H. N. W. Lekkerkerker, Phys. Rev. Lett. 95, 077801 (2005).

[10] D. van der Beek, A. V. Petukhov, P. Davidson, J. Ferré, J. P. Jamet, H. H. Wensink, G. J. Vroege, W. Bras, and H. N. W. Lekkerkerker, Phys. Rev. E 73, 041402 (2006).

[11] A. R. Khokhlov and A. N. Semenov, Macromolecules 15, 1272 (1982); S. Varga, G. Kronome, and I. Szalai, Mol. Phys. 98, 911 (2000).

[12] J. Tang and S. Fraden, Phys. Rev. Lett. 71, 3509 (1993).

[13] M. R. Kuzma, Phys. Rev. Lett. 57, 349 (1986).

[14] N. E. Geacintov, F. van Nostrand, M. Pope, and J. B. Tinkel, Biochim. Biophys. Acta 226, 486 (1971).

[15] C. P. Fan and M. J. Stephen, Phys. Rev. Lett. 25, 500 (1970).

[16] H. H. Wensink and G. J. Vroege, Phys. Rev. E 72, 031708 (2005).

[17] As was noted before in an excellent book on the history of liquid crystal science [T. J. Sluckin, D. A. Dunmur, and H. Stegemeyer, Crystals that Flow, The Liquid Crystals Book Series (Taylor \& Francis, London, 2004)], there are a number of different spellings in literature of the name of Vsevolod Konstantinovich Frederiks. In our paper, except for one reference, we will stick to the modern English phonetic transliteration of his name. Note that in Russian there is no ambiguity at all.

[18] A. Repiova and V. Frederiks, J. Russ. Phys.-Chem. Soc. 59, 183 (1927); V. Fréedericksz and V. Zolina, Trans. Am. Electrochem. Soc. 55, 85 (1929).

[19] P. G. de Gennes, The Physics of Liquid Crystals (Clarendon
Press, Oxford, 1974).

[20] A. J. Hurd, S. Fraden, F. Lonberg, and R. B. Meyer, J. Phys. (Paris) 46, 905 (1985); B. J. Lemaire, P. Davidson, D. Petermann, P. Panine, I. Dozov, D. Stoenescu, and J. P. Jolivet, Eur. Phys. J. E 13, 309 (2004).

[21] Z. Dogic and S. Fraden, Langmuir 16, 7820 (2000).

[22] E. Carr, Mol. Cryst. Liq. Cryst. 34, 159 (1977).

[23] E. Guyon, R. Meyer, and J. Salan, Mol. Cryst. Liq. Cryst. 54, 261 (1979).

[24] A. M. Wierenga, T. A. J. Lenstra, and A. P. Philipse, Colloids Surf., A 134, 359 (1998); D. van der Beek and H. N. W. Lekkerkerker, Langmuir 20, 8582 (2004).

[25] F. M. van der Kooij, D. van der Beek, and H. N. W. Lekkerkerker, J. Phys. Chem. B 105, 1696 (2001).

[26] D. G. A. L. Aarts, J. H. van der Wiel, and H. N. W. Lekkerkerker, J. Phys.: Condens. Matter 15, S245 (2003).

[27] J. E. G. J. Wijnhoven, D. D. van 't Zand, D. van der Beek, and H. N. W. Lekkerkerker, Langmuir 21, 10422 (2005).

[28] G. H. Koenderink, D. G. A. L. Aarts, V. W. A. de Villeneuve, A. P. Philipse, R. Tuinier, and H. N. W. Lekkerkerker, Biomacromolecules 4, 129 (2003).

[29] D. van der Beek, H. Reich, P. van der Schoot, M. Dijkstra, T. Schilling, R. Vink, M. Schmidt, R. van Roij, and H. Lekkerkerker, Phys. Rev. Lett. 97, 087801 (2006).

[30] M. Impéror-Clerc and P. Davidson, Eur. Phys. J. B 9, 93 (1999).

[31] B. J. Lemaire, P. Panine, J. C. P. Gabriel, and P. Davidson, Europhys. Lett. 59, 55 (2002).

[32] H. Saalfeld and M. Wedde, Z. Kristallogr. 139, 129 (1974).

[33] S. M. Clarke, A. R. Rennie, and P. Convert, Europhys. Lett. 35, 233 (1996).

[34] K. R. Purdy, Z. Dogic, S. Fraden, A. Rühm, L. Lurio, and S. G. J. Mochrie, Phys. Rev. E 67, 031708 (2003).

[35] M. Bier, L. Harnau, and S. Dietrich, Phys. Rev. E 69, 021506 (2004).

[36] Z. Dogic, J. Zhang, A. W. C. Lau, H. Aranda-Espinoza, P. Dalhaimer, D. E. Discher, P. A. Janmey, R. D. Kamien, T. C. Lubensky, and A. G. Yodh, Phys. Rev. Lett. 92, 125503 (2004); 93, 219902(E) (2004).

[37] G. Srajer, S. Fraden, and R. B. Meyer, Phys. Rev. A 39, 4828 (1989).

[38] S. Fraden and R. B. Meyer, Phys. Rev. Lett. 57, 3122 (1986).

[39] F. Lonberg, S. Fraden, A. J. Hurd, and R. Meyer, Phys. Rev. Lett. 52, 1903 (1984); C. R. Fincher, Macromolecules 19, 2431 (1986); M. Grigutsch, N. Klöpper, H. Schmiedel, and R. Stannarius, Phys. Rev. E 49, 5452 (1994). 